\section{Call from China for joint nanotech toxicity-testing effort}

SIR - In response to your News story 'Nanoparticle safety in doubt' about lung damage in Chinese factory workers (Nature 460, 937; 2009), we would like to stress that China has been paying close attention to research into and documentation of the risks of working with nanomaterials.

As in most Western countries, industrial use of nanoscale products has been proliferating in China over the past decade. These are widely used in weaving, dyeing, cosmetics and medicine, for example, as well as in waste-water treatments. The need to develop international standard-analysis protocols to assess nanoparticle toxicities is therefore becoming increasingly urgent.

China is taking measures to address the issues (for a review, see G. Q. Zhou et al. Prog. Biochem. Biophys. 35, 998-1006; 2008). These have included a series of national conferences and symposia on the safety of nanomaterials: for example, the 243rd Xiangshan conference in 2004. Research projects such as the ' 973 programme' of basic research and development have been initiated by the Chinese ministry of science and technology. And several important projects have been funded by the National Natural Science Foundation.

A recently established Chinese journal, Asian Journal of Ecotoxicology, publishes primary research papers and reviews on nanoparticle safety and toxicity in almost every issue. There are regular updates on developments in the field and a scientific platform for data sharing and policy discussion (for example, see N. Wang et al. Asian J. Ecotoxicol. 2, 252-264; 2007).

We urge the relevant international scientific organizations to join forces and work out a scheme for establishing high-speed tests that will sort out safety and toxicity issues for different industrial nanomaterials (R. F. Service Science 321, 1036-1037; 2008).

Such a collaborative venture would accelerate progress in this rapidly expanding field.

Shuping Bi, Jing Zhang, Jiongjia Cheng Chemistry Department, Nanjing University, Nanjing 210093, China e-mail: bisp@nju.edu.cn

\section{Consent: criteria should be drawn up for tissue donors}

SIR - The drive to develop new human pluripotent stem-cell lines has attracted a new, exuberant cohort of researchers who may not be familiar with the regulations and standards governing donation of human tissue. Scientists should ask donors to agree to some basic rules for research involving induced pluripotent stem (iPS) cell lines derived from their tissue. This would help to prevent road blocks of the kind highlighted in your Editorial (Nature 460, 933; 2009) about Sabine Conrad and colleagues' Corrigendum to their paper 'Generation of pluripotent stem cells from adult human testis' (Nature 460, 1044; 2009).

The rules would cover sharing cell lines with other investigators, as in this case; carrying out large-scale genome sequencing; injecting iPS cells or their derivatives into animals; and patenting discoveries or commercial uses arising from iPS cells or derivatives, with no sharing of royalties with donors. Additional specific consent would be needed for allogeneic human transplantation or reproductive research using gametes derived from iPS cells. Permission might be needed to recontact donors about new research proposals.

The advent of human adult germline stem cells and iPS cells avoids ethical issues over the use of early human embryos. So, unlike human embryonic stem cells, there is not likely to be a shortage of donors for germline stem cells or iPS cells (except in the case of rare diseases). Given that iPS cell lines can be propagated indefinitely, they are likely to become widely used. But applications still require proper consent from the tissue donor.

The proposed minimumconsent criteria from tissue donors should help to maximize the scientific value of cell lines in realizing the promise of this technology.

\section{Bernard Lo Program in Medical} Ethics and Department of Medicine, University of California, San Francisco, California 94143, USA e-mail: bernard.lo@ucsf.edu

Bruce R. Conklin Gladstone Institute of Cardiovascular Disease and Departments of Medicine and Molecular Pharmacology,

University of California, San Francisco

\section{Consent: a need for guidelines to reflect local considerations}

SIR - As you point out in your Editorial (Nature 460, 933; 2009) on the distribution of human cell lines, withholding scientific material from the broader research community contravenes the basic norms of science. We do not believe, however, that standard international consent guidelines for tissue donors are the solution to this problem, and suggest that these should instead be devised on a local scale, in collaboration with ethics committees.

Far from research being "hindered by restrictions from donors" as you suggest, people are generally willing to donate tissue for research, and even to give open-ended consent to unspecified future applications. This willingness is underpinned by donors' faith in medical research and in their right to protection and confidentiality; the assumption is that their tissue will be used only for 'ethical' investigations.

But problems can arise, for example, over whether consent covers the proposed usage (at present there are many different models of consent, ranging from specific to general), and when and how tissue should be discarded (K. Aalto-Setälä et al. PLoS Biol. 7, e42; 2009).

The answers may not always be obvious, and ethics committees (in collaboration with donors or their representatives) need to take into account the type of tissue involved, as well as the demographics and potential vulnerability of the donor or donor community, in judging the acceptability of a research proposal.

None of this precludes distribution of tissue in the name of scientific progress, nor should it if the wishes of donors are to be respected. However, it does challenge any unqualified presumption among researchers about access to human material; it also calls into question the ethical acceptability of using internationally standardized consent forms, as recommended in your Editorial. Rather, we would argue for international standards to ensure that tissue distribution is not thwarted by ethics committees, accompanied by a plurality of local approaches to obtaining consent.

This strategy would address the problems you outline, while demonstrating respect for moral decisions made by individuals and groups and preserving donors' trust in biological medicine.

Wendy Lipworth, Rob Irvine, Bronwen Morrell Centre for Values, Ethics and the Law in Medicine, Medical Foundation Building, University of Sydney, NSW 2006, Australia e-mail:w.lipworth@usyd.edu.au

Contributions may be sent to correspondence@nature.com. Please see the Guide to Authors at http://tinyurl.com/373jsv. 